\title{
Mejoras cognitivas y perceptivo-motrices en personas mayores participantes en un programa de estimulación integral cognitivo- motriz
}

Cognitive and perceptual-motor improvements in elderly participating in a cognitive-motor integrated stimulation program

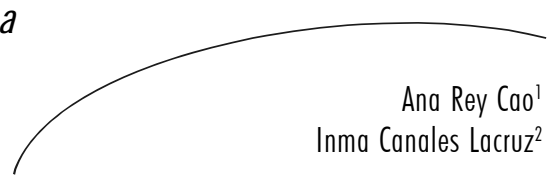

Resumo

Este artículo tiene como objetivo mostrar los efectos cognitivos y perceptivomotrices obtenidos tras la aplicación de un programa de estimulación cognitiva a través de la motricidad para personas mayores llamado "Memoria en Movimiento". Se trata de una investigación cuasi-experimental en la que participaron 234 personas mayores con una media de edad de $69.92 \pm 7.23$ distribuidas en grupo experimental inactivo físicamente y grupo control praticantes de Tai-chi y Gimnasia. La evaluación se realizó antes de la aplicación del programa y tras su finalización. Se utilizaron el Mini Examen Cognoscitivo, el Test de Dígitos - Total, Directo e Inverso -, una Prueba de Memoria Motriz y una Prueba de Orientación Espacial - Total, 1 y 2 -. Se constató una mejoría significativa $(\leq 0.05)$ del grupo experimental en todas las mediciones de estudio excepto en Dígitos Directo y Orientación Espacial 2. El grupo control consigue mejoras significativas $(\leq 0.05)$ exclusivamente en Orientación Espacial Total y Espacial $1 \mathrm{y}$ deterioro significativo $(\leq 0.05)$ en Memoria Motriz. El programa "Memoria en Movimiento" mejora la atención, la memoria, la conciencia corporal y la estructuración espacial de las personas mayores mediante tareas cognitivas con implicación motriz integral.

\section{Abstract}

This paper aims to show the cognitive and perceptual-motor effects obtained after the application of a cognitive stimulation program through the motricity for the elders called "Memory in Movement". This is a quasi-experimental research in which participated 234 major persons by an average of age of 69.92

\footnotetext{
Universidad de Vigo. Departamento de Didácticas Especiales. Facultad de Ciencias de la Educación y del Deporte. Pontevedra, España.

2 Universidad de Zaragoza. Departamento de Expresión plástica, musical y corporal. Área Didáctica de la Expresión Corporal. Facultad de Educación. Zaragoza, España.

Correspondência / Correspondence

Ana Rey Cao

Facultad de Ciencias da Educación e do Deporte. Universidad de Vigo. Campus A Xunqueira s/n. 36005 Pontevedra (España).

E-mail: anacao@uvigo.es
}

Palabras clave: Memoria. Movimiento. Cognitivo. Envejecimiento. Autoestimulación. Anciano. 
\pm 7.23 , distributed between experimental group inactive physically and the control group practice Tai-chi and gymnastics. The evaluation was made before application of the program and after its conclusion. It was used the Mini Cognitive Test, the WAIS-R Digit Span Test Direct and Inverse, a Test of Motor Memory and a Spatial Orientation test-Total, 1 and 2. A significant improvement $(\leq 0.05)$ was checked in the experimental group in all the measurements of study excepting Digits Direct and Spatial Orientation 2. The control group achieved significant improvements $(\leq 0.05)$ exclusively in Spatial Orientation Total and Spatial 1 and it got significant deterioration $(\leq 0.05)$ in Motor Memory. The program "Memory in Movement" improves the attention, the memory, the body awareness and the spatial structuring of the elders by means of cognitive tasks with integral motor implication.
Key words: Memory. Movement. Cognitive. Aging. Self Stimulation. Aged.

\section{INTRODUCCIÓN}

El envejecimiento conlleva alteraciones en las funciones cognitivas, ${ }^{1,2}$ físicas $^{3}$ y/o perceptivomotrices. ${ }^{4}$ El grado de deterioro se relaciona con los niveles de práctica habitual de estas funciones, por lo que una intervención sistemática puede ayudar a contrarrestarlo. ${ }^{5,6}$

El entrenamiento cognitivo mejora las funciones cognitivas. ${ }^{7,8}$ Del mismo modo, la práctica de ejercicio físico se asocia a mejoras cognitivas, ${ }^{9}$ físicas ${ }^{10}$ y perceptivo-motrices. ${ }^{4}$

Algunas intervenciones integran el entrenamiento cognitivo y físico. Es el caso de "Motricidad y memoria", "Memory Workout Program", ${ }^{12}$ "Vivir con vitalidad" y el programa mental y aeróbico de Fabre et al. ${ }^{13}$

Concretamente, la investigación de Fabre et al. ${ }^{13}$ compara los efectos de un entrenamiento aeróbico, un entrenamiento mental y la combinación de ambos sobre las funciones cognitivas. Concluye que su combinación consigue mayores efectos cognitivos que cualquiera de los entrenamientos por sí solos.

Pilar Pont ${ }^{11}$ aplica una intervención que integra tareas perceptivo-motrices y de trabajo aeróbico para el entrenamiento de la memoria a partir de la vivencia corporal del movimiento. Esta investigadora demuestra que el trabajo cognitivo y psicomotriz integrado en una única intervención es más efectivo que su trabajo por separado.
Dado que el envejecimiento sensoriomotor y cognitivo están causalmente relacionados y son funcionalmente interdependientes, ${ }^{14,15}$ la combinación de trabajo perceptivo-motriz y cognitivo en tareas que requieren elevado control ejecutivo podría tener recíprocas transferencias positivas. Además los efectos positivos del ejercicio físico sobre la cognición son mayores si los procesos implicados requieren un elevado control ejecutivo. ${ }^{16}$

El componente motriz de los programas de entrenamiento cognitivo-motriz integrado no está centrado en el trabajo físico-condicional, como es el caso de las investigaciones que evalúan fundamentalmente la relación entre el ejercicio físico y la mejora cognitiva utilizando métodos básicamente aeróbicos y/o calistenias, ${ }^{9}$ sino que incorporan tareas perceptivo-motrices en las que lo relevante son las operaciones cognitivas que deben realizarse para su resolución.

En esta línea y con la intención de constituir un programa integral cognitivo-motriz denominado "Memoria en Movimiento", ${ }^{17}$ nació en el año 2007 el proyecto de Investigación + Desarrollo - CO-015-07 - denominado "Estimulación cognitiva a través de la motricidad para personas mayores" - ECAM -. Ha sido desarrollado por el grupo de Investigación DE-4 de la Facultad de Ciencias de la Educación y del Deporte de la Universidad de Vigo (España) y financiado por la Obra Social Caixa Galicia (España). 
Por tanto, es objetivo de este artículo mostrar las mejoras cognitivas y perceptivo motrices en las personas mayores que han participado en el programa integral cognitivo-motriz "Memoria en Movimiento"*.

\section{MÉTODO}

\section{Participantes}

Participaron 234 personas con una media de edad de $69.92 \pm 7.23$ procedentes de ocho Centros de Mayores de diferentes núcleos urbanos de la comunidad autónoma gallega (España), distribuidas en grupo experimental $(\mathrm{n}=185)$ y grupo control $(\mathrm{n}=49)$. La disparidad de $n$ entre los dos grupos se debe a que la investigación se realiza en contextos reales con grupos naturales. ${ }^{18}$

Los criterios de selección fueron: edad superior a 60 años, ausencia de deterioro cognitivo; inexistencia de problemas de movilidad y no institucionalización.

Para la recogida y utilización confidencial de los datos se solicitó su consentimiento informado.

Tabla 1- Características de las personas participantes. Pontevedra, España, 2010.

\begin{tabular}{lcccrcrr}
\hline & & \multicolumn{2}{c}{$\%$ Sexo } & \multicolumn{2}{c}{ Edad (años) } & \multicolumn{2}{c}{ n por intervalos de edad } \\
\cline { 3 - 8 } & & Hombres & Mujeres & Media \pm D.T. & $60-69$ & $70-79$ & $80-89$ \\
\hline Grupo e & 185 & 17.3 & 82.7 & $69.94 \pm 7.54$ & 78 & 87 & 20 \\
Grupo c & 49 & 24.5 & 75.5 & $69.86 \pm 5.99$ & 27 & 18 & 4 \\
Total & 234 & 18.8 & 81.2 & $73.37 \pm 7.90$ & 105 & 105 & 24 \\
\hline
\end{tabular}

Grupo e: grupo experimental; Grupo c: grupo control.

\section{Procedimiento}

Mediante un diseño cuasi-experimental se aplicó "Memoria en Movimiento" al grupo experimental y se evaluaron los resultados en los dos grupos control y experimental - antes y después del programa - cuatro meses del año 2008 -. El grupo control participaba en otros programas de actividad física ofertados por el propio Centro de Mayores - Tai-chi y Gimnasia -, los cuales no eran regulados por el propio grupo de investigación.

\section{Análisis estadístico}

Se realizó un análisis descriptivo y un análisis inferencial. En este último, debido a la no normalidad de la muestra - Kolmogorov-Smirnov; $\mathrm{p}<0.05-$, se utilizaron pruebas no paramétricas: pares relacionados de Wilcoxon y $\mathrm{H}$ de KruskalWallis, estimándose significativo $\leq 0.05$.

\section{Variables dependientes}

Deterioro cognitivo, memoria de trabajo, memoria inmediata, memoria motriz, atención, conciencia corporal y estructuración espacial.

\section{Variable independiente}

El programa de intervención "Memoria en Movimiento", ${ }^{17}$ caracterizado por la implicación de la motricidad en la resolución de tareas que requieren de elevado control ejecutivo.

\footnotetext{
El programa "Memoria en Movimiento" se puede descargar de forma gratuita en el siguiente enlace de la Obra Social Caixa Galicia: http://www.obrasocialncg.com/noticias/mostrar/publicaciones_sobre_dependencia_envejecimiento_ activo_y_estimulacion_cognit.
} 
Está organizado en cuatro unidades didácticas, con un total de 26 sesiones de una hora de duración y 120 tareas en torno a cuatro bloques de contenidos: conciencia corporal, atención, estrategias para recordar y estructuración espaciotemporal. La frecuencia de aplicación es de dos sesiones semanales.

Las tareas propuestas respetan las particularidades perceptivas, motrices y motivacionales del envejecimiento. Estas características hacen necesario que las tareas se adapten al enlentecimiento de la velocidad de procesamiento, ya que suele afectar notablemente y de forma generalizada a las personas mayores.

En consecuencia, los problemas derivados de la falta de atención y concentración - función inhibitoria -, y los derivados de la entrada de la información - enlentecimiento de la velocidad de procesamiento y problemas en los órganos de los sentidos -, son los principales aspectos que determinan el análisis de la aplicabilidad del programa. Además se evitan aquellas tareas complejas en las que los elementos novedosos a procesar son más de uno, ya que obtienen peores resultados en la compresión y la participación. ${ }^{19}$

Tabla 2 - Contenidos y sesiones de las Unidades Didácticas de "Memoria en Movimiento". Pontevedra, España, 2010.

\begin{tabular}{lllll}
\hline & \multicolumn{1}{c}{$\begin{array}{c}\text { Unidad 1: Soy } \\
\text { Corpóreo }\end{array}$} & $\begin{array}{c}\text { Unidad 2: Cuerpo } \\
\text { atento }\end{array}$ & $\begin{array}{c}\text { Unidad 3: Cuerpo } \\
\text { que recuerda }\end{array}$ & $\begin{array}{c}\text { Unidad 4: Cuerpo } \\
\text { orientado }\end{array}$ \\
\hline $\mathrm{N}^{\circ}$ sesiones & \multicolumn{1}{c}{5} & 6 & 8 & 7 \\
$\begin{array}{l}\text { Contenidos } \\
\text { fundamentales }\end{array}$ & $\begin{array}{l}\text { Respiración y } \\
\text { relajación } \\
\text { Movilidad articular }\end{array}$ & $\begin{array}{l}\text { Atención auditiva } \\
\text { Atención visual } \\
\text { Atención } \\
\text { Elongación }\end{array}$ & $\begin{array}{l}\text { Estrategias de } \\
\text { memoria } \\
\text { Técnicas de } \\
\text { memoria }\end{array}$ & $\begin{array}{l}\text { Estructuración } \\
\text { espacial } \\
\text { Esquema corporal }\end{array}$ \\
& & & $\begin{array}{l}\text { Estructuración } \\
\text { temporal } \\
\text { Estructuración } \\
\text { espacio-temporal }\end{array}$ \\
\hline
\end{tabular}

La Unidad Didáctica 1 "Soy Corpóreo" optimiza el conocimiento del propio cuerpo mediante la realización de tareas corporales que inciden en la conciencia, imagen y esquema corporal. Introduce el control respiratorio como estrategia que predispone a la concentración y a la relajación. Se realizan tareas sencillas dónde el alumnado memoriza secuencias de respiración y rutinas de movilidad articular.

La Unidad Didáctica 2 "Cuerpo atento" estimula la sensibilidad exteroceptiva de los órganos de la visión y la audición y posteriormente la atención ante informaciones espaciales y temporales que, en algunas actividades, han sido memorizadas previamente por los alumnos/as. También trabaja la sensibilidad propioceptiva, estimulando la atención a las informaciones que provienen de nuestro propio cuerpo - músculos, tendones, huesos y articulaciones.

La Unidad Didáctica 3 "Cuerpo que recuerda" está destinada al aprendizaje y práctica de las estrategias mnésicas de la repetición, la visualización y la asociación; y de las técnicas mnésicas de pares asociados, método de la cadena, método Loci, método del agrupamiento y método de la historia. El aprendizaje se realiza aplicando estos recursos cognitivos en tareas motrices que requieren de la memorización de diferentes elementos tales como movimientos, posturas, objetos, trayectorias, ritmos, etc. 
Por último, la Unidad Didáctica 4 "Cuerpo orientado" trabaja la exterognosia, es decir, el conocimiento y adecuación a las coordenadas espacio-temporales. La expansión de la conciencia espacial se realiza - partiendo de la consolidación de la conciencia corporal iniciada en la unidad didáctica 1 - a través de tareas que incorporan la locomoción. Los ejercicios incluyen desplazamientos en los que deben realizarse estimaciones en función de referencias del espacio exterior y/o memorizaciones de trayectorias. Finalmente se incorporan desplazamientos que requieren la interpretación de representaciones topográficas. La conciencia temporal se trabaja con ejercicios de adecuación del movimiento que necesitan de la percepción de la duración y el orden. Los alumnos/as memorizan y ejecutan motrizmente diversidad de secuencias rítmicas.

\section{Instrumentos}

Se utilizaron tres herramientas: una evaluación informal, un informe médico y una evaluación formal.

En la evaluación informal, los profesionales de los centros aplicaron un protocolo de observación a través del cual identificaron a potenciales alumnos/as. Este protocolo está compuesto por dos partes. La primera parte adaptada del estadiaje evolutivo Functional Assesment Stagin-FAST $-{ }^{20}$ destinada a identificar a personas mayores sin deterioro cognitivo. La segunda parte destinada a determinar los dominios de los potenciales alumnos/as con respecto a su movilidad basándose en criterios de la Clasificación Internacional del Funcionamiento de la Discapacidad y de la Salud - CIF - ${ }^{21}$.

Posteriormente las personas seleccionadas que deseaban realizar el programa solicitaron a su médico un informe de idoneidad sobre su capacitación física.

La evaluación formal se centró en el Mini Examen Cognoscitivo - MEC - de 35 ítems, ${ }^{22}$ utilizando como corte para detectar deterioro cognitivo $23 / 24$ puntos.

Para la evaluación de los efectos del programa se administraron: el MEC para evaluar el funcionamiento cognitivo general; el Test de Dígitos -WAIS III - ${ }^{23}$ que consta de una prueba directa e inversa y evalúa la atención, la memoria inmediata y la memoria de trabajo; una prueba de Memoria Motriz ${ }^{17}$ que consiste en la reproducción de diez posturas estáticas para evaluar la memoria motriz y la conciencia corporal; y una prueba de Orientación Espacial adaptada de Feuerstein ${ }^{24}$ que consiste en recorrer ocho trayectorias identificando las direcciones prueba de Orientación Espacial 1 - y los puntos cardinales - prueba de Orientación Espacial 2. ${ }^{17}$

El diseño de investigación fue aprobado por el Comité de Ética de la Obra Social Caixa Galicia y sigue los principios éticos de la Declaración de Helsinki de la Asociación Médica Mundial que rigen las investigaciones biomédicas con personas.

\section{RESULTADOS}

De las puntuaciones pretest - tabla 3 destacan las diferencias significativas $(\leq 0.05)$ en Memoria Motriz. El grupo experimental tiene una media de $5.62 \pm 2.6$, mientras que el grupo control consigue una media de $8.16 \pm 1.9$, casi 3 puntos de diferencia en una puntuación máxima de 10. En el resto de valores iniciales no se aprecian diferencias significativas $(\geq 0.05)$ entre ambos grupos. 
Tabla 3 - Análisis descriptivo e inferencia estadística intergrupos de las puntuaciones obtenidas en pre test. Pontevedra, España, 2010.

\begin{tabular}{lcccc}
\hline & \multicolumn{2}{c}{ Media \pm D.T. } & \multicolumn{2}{c}{ P. Wilcoxon } \\
\hline & $\begin{array}{c}\text { Grupo e } \\
(\mathrm{n}=185)\end{array}$ & $\begin{array}{c}\text { Grupo c } \\
(\mathrm{n}=49)\end{array}$ & $\mathrm{Z}$ & $\begin{array}{c}\text { Valor de } \\
\text { Significación }\end{array}$ \\
\cline { 2 - 5 } MEC (max. 35 puntos) & $30.91 \pm 3.5$ & $31.73 \pm 2.4$ & -1.217 & 0.224 \\
Dígitos Total (max. 30 puntos) & $11.50 \pm 3.3$ & $11.00 \pm 2.9$ & -0.906 & 0.365 \\
Dígitos Directo (max.16 puntos) & $7.15 \pm 1.9$ & $6.76 \pm 1.7$ & -1.425 & 0.154 \\
Dígitos Inverso (max. 14 puntos) & $4.39 \pm 1.9$ & $4.18 \pm 1.6$ & -0.372 & 0.710 \\
P. Memoria Motriz (max. 10 puntos) & $5.62 \pm 2.6$ & $8.16 \pm 1.9$ & -6.073 & $0.001^{*}$ \\
P. Orientac. Esp. Total (max. 16 puntos) & $8.82 \pm 4.2$ & $9.12 \pm 4.8$ & -0.778 & 0.437 \\
P. Orientación Espacial 1 (max. 8 puntos) & $5.03 \pm 2.1$ & $4.76 \pm 2.5$ & -0.372 & 0.710 \\
P. Orientación Espacial 2 (max. 8 puntos) & $4.02 \pm 2.6$ & $4.37 \pm 2.6$ & -1.328 & 0.184 \\
\hline
\end{tabular}

Grupo e: Grupo experimental; Grupo c: Grupo control $\mathrm{p}<0.05 *$ (La diferencia es significativa al nivel de 0.05)

En las tablas 4 y 5 se presentan las medias y desviaciones típicas, así como los resultados del análisis inferencial a partir de la prueba de Wilcoxon del grupo experimental y control.

Tabla 4 - Análisis descriptivo e inferencia estadística de las puntuaciones obtenidas en pre-post test del grupo experimental. Pontevedra, España, 2010.

\begin{tabular}{lcccc}
\hline \multicolumn{5}{c}{ Grupo experimental (n=185) } \\
\cline { 2 - 5 } & \multicolumn{2}{c}{ Media \pm D.T. } & \multicolumn{2}{c}{ P. Wilcoxon } \\
\cline { 2 - 5 } & Pretest & Postest & Z & $\begin{array}{c}\text { Valor de } \\
\text { Significación }\end{array}$ \\
\cline { 2 - 5 } MEC (max. 35 puntos) & $30.91 \pm 3.5$ & $32.41 \pm 2.8$ & -7.649 & $0.001^{*}$ \\
Dígitos Total (m ax. 30 puntos) & $11.5 \pm 3.3$ & $12.09 \pm 3.3$ & -4.130 & $0.001 *$ \\
Dígitos Directo (max.16 puntos) & $7.15 \pm 1.9$ & $7.3 \pm 1.9$ & -1.860 & 0.063 \\
Dígitos Inverso (max. 14 puntos) & $4.39 \pm 1.9$ & $4.76 \pm 1.8$ & -3.549 & $0.001 *$ \\
P. Memoria Motriz (max. 10 puntos) & $5.62 \pm 2.6$ & $7.08 \pm 2.6$ & -6.709 & $0.001^{*}$ \\
P. Orienta c. Esp. Total (max. 16 puntos) & $8.82 \pm 4.2$ & $11.81 \pm 3.7$ & -8.685 & $0.001^{*}$ \\
P. Orientación Espacial 1 (max. 8 puntos) & $5.03 \pm 2.1$ & $6.36 \pm 1.7$ & -7.222 & $0.001^{*}$ \\
P. Orientación Espacial 2 (max. 8 puntos) & $4.02 \pm 2.6$ & $5.65 \pm 2.1$ & -7.103 & 0.240 \\
\hline
\end{tabular}

$\mathrm{p}<0.05 *$ (La diferencia es significativa al nivel de 0.05$)$

La tabla 4 refleja las mejoras significativas $(\leq 0.05)$ del grupo experimental en todos los valores excepto en el Dígitos Directo y
Orientación Espacial 2. En estas dos pruebas las mejoras no son significativas $(\geq 0.05)$. 
Tabla 5 - Análisis descriptivo e inferencia estadística de las puntuaciones obtenidas en pre-post test del grupo control. Pontevedra, España, 2010.

\begin{tabular}{lcccc}
\hline \multicolumn{4}{c}{ Grupo control (n=49) } \\
\cline { 2 - 5 } & \multicolumn{2}{c}{ Media \pm D.T. } & \multicolumn{2}{c}{ P. Wilcoxon } \\
\cline { 2 - 5 } & Pretest & Postest & Z & $\begin{array}{c}\text { Valor de } \\
\text { Significación }\end{array}$ \\
\cline { 2 - 5 } MEC (max. 35 puntos) & $31.73 \pm 2.4$ & $31.59 \pm 3.2$ & -0.072 & 0.942 \\
Dígitos Total (max. 30 puntos) & $11.00 \pm 2.9$ & $11.08 \pm 2.4$ & -0.413 & 0.680 \\
Dígitos Directo (max.16 puntos) & $6.76 \pm 1.7$ & $6.76 \pm 1.7$ & -0.105 & 0.916 \\
Dígitos Inverso (max. 14 puntos) & $4.18 \pm 1.6$ & $4.29 \pm 1.1$ & -0.576 & 0.565 \\
P. M emoria Motriz (max. 10 puntos) & $8.16 \pm 1.9$ & $6.90 \pm 2.5$ & -4.325 & $0.001 *$ \\
P. Orientac. Esp. Total (max. 16 puntos) & $9.12 \pm 4.8$ & $10.31 \pm 4.0$ & -3.327 & $0.001 *$ \\
P. Orientación Espac. 1 (max. 8 puntos) & $4.76 \pm 2.5$ & $5.65 \pm 1.6$ & -2.808 & $0.005^{*}$ \\
P. Orientación Espac. 2 (max. 8 puntos) & $4.37 \pm 2.6$ & $4.65 \pm 2.7$ & -1.176 & 0.240 \\
\hline
\end{tabular}

$\mathrm{p}<0.05 \%$ (La diferencia es significativa al nivel de 0.05$)$

La tabla 5 revela mejoras significativas $(\leq$ 0.05) del grupo control en Orientación Espacial Total y Espacial 1 y deterioro significativo $(\leq 0.05)$ en Memoria Motriz. No se producen mejoras significativas en el resto de los valores.

Tabla 6 - Análisis descriptivo e inferencia estadística de las puntuaciones obtenidas en pre test del grupo experimental según intervalos de edad. Pontevedra, España, 2010.

\begin{tabular}{lccccc}
\hline \multicolumn{5}{c}{ Grupo experimental $(\mathrm{n}=185)$} \\
\hline & $\begin{array}{c}50-69 \\
(\mathrm{n}=78)\end{array}$ & $\begin{array}{c}70-79 \\
(\mathrm{n}=87)\end{array}$ & $\begin{array}{c}80-89 \\
(\mathrm{n}=20)\end{array}$ & Chi $^{2}$ & $\begin{array}{c}\text { Valor de } \\
\text { Significación }\end{array}$ \\
\cline { 2 - 6 } MEC & $31.66 \pm 3.0$ & $30.09 \pm 3.6$ & $31.40 \pm 4.2$ & 11.205 & $0.004^{*}$ \\
Dí́itos Total & $11.86 \pm 3.2$ & $11.01 \pm 3.2$ & $12.15 \pm 3.6$ & 2.094 & 0.351 \\
Dígitos Directo & $7.31 \pm 2.0$ & $6.89 \pm 1.7$ & $7.65 \pm 1.8$ & 2.649 & 0.266 \\
Dígitos Inverso & $4.70 \pm 2.0$ & $4.12 \pm 1.7$ & $4.30 \pm 1.8$ & 2.408 & 0.300 \\
P. Memoria Motriz & $5.62 \pm 8.4$ & $5.52 \pm 2.4$ & $6.00 \pm 2.5$ & 8.781 & $0.012^{*}$ \\
P. Orientac. Esp. Total & $9.74 \pm 4.1$ & $7.97 \pm 4.1$ & $9.25 \pm 4.8$ & 13.903 & $0.001^{*}$ \\
P. Orientac. Espacial 1 & $5.53 \pm 2.1$ & $4.55 \pm 2.0$ & $5.44 \pm 2.3$ & 3.834 & 0.147 \\
P. Orientac. Espacial 2 & $4.63 \pm 2.5$ & $3.66 \pm 2.6$ & $3.88 \pm 2.9$ & 23.769 & $0.001^{*}$ \\
\hline
\end{tabular}

$\mathrm{p}<0.05 *$ (La diferencia es significativa al nivel de 0.05 )

Los datos de la tabla 6 no permiten establecer una relación directa edad-rendimiento del grupo experimental, ya que los sujetos más mayores -80 89 años - consiguen mejores rendimientos que los sujetos incluidos en el segundo intervalo de edad -
70-79 años. No obstante, se debe tener en cuenta la desproporción de $\mathrm{n}$ del tercer intervalo de edad con respecto a los otros dos. En la mayoría de las puntuaciones los sujetos más jóvenes - 60-69 años - consiguen los mejores rendimientos. 
La prueba $\mathrm{H}$ de Kruskal-Wallis constata diferencias significativas $(\leq 0.05)$ según intervalos de edad en MEC, Memoria Motriz, Orientación Espacial Total y Espacial 2.

Tabla 7 - Análisis descriptivo e inferencia estadística de las puntuaciones obtenidas en pre-post test del grupo experimental según intervalos de edad. Pontevedra, España, 2010.

\begin{tabular}{|c|c|c|c|c|c|c|}
\hline \multicolumn{7}{|c|}{ Grupo experimental $(n=185)$} \\
\hline & & & \multirow[b]{2}{*}{ Pretest } & \multirow[b]{2}{*}{ Postest } & \multicolumn{2}{|c|}{ P. Wilcoxon } \\
\hline & & & & & Z & $\begin{array}{c}\text { Valor de } \\
\text { Significación }\end{array}$ \\
\hline & MEC & Media \pm D.T. & $31.66 \pm 3.0$ & $33.04 \pm 2.4$ & -5.291 & $0.001 \%$ \\
\hline & Dig. Tot. & Media \pm D.T. & $11.86 \pm 3.2$ & $12.44 \pm 3.0$ & -2.580 & $0.010 *$ \\
\hline & Dig. Dir. & Media \pm D.T. & $7.31 \pm 2.0$ & $7.39 \pm 1.8$ & -0.917 & 0.359 \\
\hline $50-69$ & Dig. In. & Media \pm D.T. & $4.70 \pm 2.0$ & $5.05 \pm 1.6$ & -1.768 & 0.077 \\
\hline \multirow[t]{7}{*}{$(\mathrm{n}=78)$} & Mem. Mot. & Media \pm D.T. & $5.62 \pm 8.4$ & $7.65 \pm 2.3$ & -5.681 & $0.001 \%$ \\
\hline & Orient. Tot. & Media \pm D.T. & $9.74 \pm 4.1$ & $12.15 \pm 4.0$ & -4.923 & $0.001 *$ \\
\hline & Orient. 1 & Media \pm D.T. & $5.53 \pm 2.1$ & $6.60 \pm 1.9$ & -3.965 & $0.001 *$ \\
\hline & Orient. 2 & Media \pm D.T. & $4.63 \pm 2.5$ & $5.8 \pm 12.1$ & -3.052 & $0.002 \%$ \\
\hline & MEC & Media \pm D.T. & $30.09 \pm 3.6$ & $31.81 \pm 2.6$ & -5.242 & $0.001 \%$ \\
\hline & Dig. Tot. & Media \pm D.T. & $11.01 \pm 3.2$ & $11.38 \pm 3.4$ & -2.044 & $0.041 *$ \\
\hline & Dig. Dir. & Media \pm D.T. & $6.89 \pm 1.7$ & $6.95 \pm 1.8$ & -0.805 & 0.421 \\
\hline 70-79 & Dig. In. & Media \pm D.T. & $4.12 \pm 1.7$ & $4.36 \pm 1.9$ & -1.748 & 0.081 \\
\hline \multirow[t]{7}{*}{$(\mathrm{n}=87)$} & Mem. Mot. & Media \pm D.T. & $5.52 \pm 2.4$ & $6.54 \pm 2.7$ & -3.612 & $0.001 \%$ \\
\hline & Orient. Tot. & Media \pm D.T. & $7.97 \pm 4.1$ & $11.52 \pm 3.4$ & -6.661 & $0.001 \%$ \\
\hline & Orient. 1 & Media \pm D.T. & $4.55 \pm 2.0$ & $6.20 \pm 1.5$ & -6.066 & $0.001 \%$ \\
\hline & Orient. 2 & Media \pm D.T. & $3.66 \pm 2.6$ & $5.54 \pm 2.1$ & -5.837 & $0.001 *$ \\
\hline & MEC & Media \pm D.T. & $31.40 \pm 4.2$ & $32.50 \pm 4.0$ & -1.910 & 0.056 \\
\hline & Dig. Tot. & Media \pm D.T. & $12.15 \pm 3.6$ & $13.70 \pm 3.6$ & -2.975 & $0.003 *$ \\
\hline & Dig. Dir. & Media \pm D.T. & $7.65 \pm 1.8$ & $8.40 \pm 2.1$ & -1.903 & 0.057 \\
\hline $80-89$ & Dig. In. & Media \pm D.T. & $4.30 \pm 1.8$ & $5.30 \pm 1.7$ & -3.272 & $0.001 \%$ \\
\hline \multirow{4}{*}{$(\mathrm{n}=20)$} & Mem. Mot. & Media \pm D.T. & $6.00 \pm 2.5$ & $7.00 \pm 3.0$ & -1.415 & 0.161 \\
\hline & Orient. Tot. & Media \pm D.T. & $9.25 \pm 4.8$ & $11.88 \pm 3.5$ & -2.516 & $0.012 \%$ \\
\hline & Orient. 1 & Media \pm D.T. & $5.44 \pm 2.3$ & $6.19 \pm 1.9$ & -1.415 & 0.157 \\
\hline & Orient. 2 & Media \pm D.T. & $3.88 \pm 2.9$ & $5.69 \pm 2.3$ & -2.945 & $0.003 \%$ \\
\hline
\end{tabular}

Dig Tot.: Dígitos Total; Dig. Dir: Dígitos Directo; Dig. In.: Dígitos Inverso; Mem. Mot.: Prueba Memoria Motriz; Orient. Tot.: Prueba Orientación Total; Orient. 1: Prueba Orientación 1; Orient. 2: Prueba Orientación 2

La tabla 7 muestra los resultados del grupo experimental según intervalos de edad. Concretamente los dos primeros intervalos de edad - 50-69 y 70-79 - obtienen mejoras significativas $(\leq 0.05)$ en todos los valores excepto en Dígitos Directo y Dígitos Inverso. Los más mayores del grupo experimental - 80-89 años obtienen mejoras significativas $(\leq 0.05)$ en cuatro de los ocho instrumentos: Dígitos Total, Dígitos Inverso; Orientación Espacial Total y Espacial 2. 
Tabla 8 - Análisis descriptivo e inferencia estadística de las puntuaciones obtenidas en pre test del grupo experimental según nivel de estudios. Pontevedra, España, 2010.

\begin{tabular}{lcccccc}
\hline \multicolumn{7}{c}{ Grupo experimental $(\mathrm{n}=185)$} \\
& \multicolumn{3}{c}{ Media \pm D.T. } \\
\cline { 2 - 7 } & $\begin{array}{c}\text { Primarios } \\
(\mathrm{n}=127)\end{array}$ & $\begin{array}{c}\text { Secundarios } \\
(\mathrm{n}=35)\end{array}$ & $\begin{array}{c}\text { Universitario } \\
(\mathrm{n}=14)\end{array}$ & $\begin{array}{c}\text { Analfabeto } \\
(\mathrm{n}=7)\end{array}$ & Chi $^{2}$ & $\begin{array}{c}\text { Valor de } \\
\text { Significación }\end{array}$ \\
\cline { 2 - 7 } MEC & $30.42 \pm 3.3$ & $33.04 \pm 2.7$ & $32.93 \pm 1.8$ & $25.21 \pm 4.0$ & 11.205 & $0.004^{*}$ \\
Dígitos Total & $10.78 \pm 2.8$ & $13.68 \pm 3.4$ & $14.14 \pm 3.4$ & $8.43 \pm 1.6$ & 2.094 & 0.351 \\
Dígitos Directo & $6.78 \pm 1.6$ & $8.38 \pm 2.0$ & $8.36 \pm 1.6$ & $5.29 \pm 1.1$ & 2.649 & 0.266 \\
Dígitos Inverso & $4.01 \pm 1.5$ & $5.47 \pm 2.3$ & $5.79 \pm 2.4$ & $3.14 \pm 1.0$ & 2.408 & 0.300 \\
Memoria Motriz & $5.20 \pm 2.5$ & $6.78 \pm 2.4$ & $6.77 \pm 2.8$ & $6.00 \pm 2.5$ & 8.781 & $0.012^{*}$ \\
Orientac. Esp. Total & $7.68 \pm 3.8$ & $12.03 \pm 3.8$ & $12.21 \pm 2.8$ & $5.67 \pm 3.0$ & 13.903 & $0.001^{*}$ \\
Orientac. Espacial 1 & $4.61 \pm 2.1$ & $6.24 \pm 1.9$ & $6.43 \pm 1.6$ & $3.83 \pm 1.4$ & 3.834 & 0.147 \\
Orientac. Espacial 2 & $3.07 \pm 2.3$ & $5.79 \pm 2.2$ & $5.86 \pm 1.9$ & $1.83 \pm 2.1$ & 23.769 & $0.001^{*}$ \\
\hline
\end{tabular}

$\mathrm{p}<0.05 *$ (La diferencia es significativa al nivel de 0.05$)$

Las medias de los valores iniciales del grupo experimental según el nivel de estudios se detallan en la tabla 8. De estos valores se desprende una relación directa nivel de estudios-rendimiento.
La prueba $\mathrm{H}$ de Kruskal-Wallis constata diferencias significativas $(\leq 0.05)$ según nivel de estudios en MEC, Memoria Motriz, Orientación Espacial Total y Espacial 2.

Tabla 9 - Análisis descriptivo e inferencia estadística de las puntuaciones obtenidas en pre-post test del grupo experimental según nivel de estudios. Pontevedra, España, 2010.

\begin{tabular}{|c|c|c|c|c|c|c|}
\hline \multicolumn{7}{|c|}{ Grupo experimental $(n=185)$} \\
\hline & & & \multirow[b]{2}{*}{ Pretest } & \multirow[b]{2}{*}{ Postest } & \multicolumn{2}{|c|}{ P. Wilcoxon } \\
\hline & & & & & $\mathrm{Z}$ & $\begin{array}{c}\text { Valor de } \\
\text { Significación }\end{array}$ \\
\hline \multirow{8}{*}{$\begin{array}{l}\text { Primarios } \\
(\mathrm{n}=127)\end{array}$} & MEC & Media \pm D.T. & $30.42 \pm 3.3$ & $32.00 \pm 2.8$ & -6.521 & $0.001 \%$ \\
\hline & Dig. Tot. & Media \pm D.T. & $10.78 \pm 2.8$ & $11.15 \pm 2.7$ & -2.713 & $0.007 *$ \\
\hline & Dig. Dir. & Media \pm D.T. & $6.78 \pm 1.6$ & $6.91 \pm 1.5$ & -1.479 & 0.139 \\
\hline & Dig. In. & Media \pm D.T. & $4.01 \pm 1.5$ & $4.24 \pm 1.5$ & -1.744 & 0.081 \\
\hline & Mem. Mot. & Media \pm D.T. & $5.20 \pm 2.5$ & $6.60 \pm 2.6$ & -5.732 & $0.001 \%$ \\
\hline & Orient. Tot. & Media \pm D.T. & $7.68 \pm 3.8$ & $10.92 \pm 3.7$ & -7.459 & $0.001 \%$ \\
\hline & Orient. 1 & Media \pm D.T. & $4.61 \pm 2.1$ & $6.04 \pm 1.7$ & -6.714 & $0.001 \%$ \\
\hline & Orient. 2 & Media \pm D.T. & $3.07 \pm 2.3$ & $5.05 \pm 2.2$ & -5.643 & $0.001 \%$ \\
\hline \multirow{8}{*}{$\begin{array}{l}\text { Secundarios } \\
(\mathrm{n}=35)\end{array}$} & MEC & Media \pm D.T. & $33.04 \pm 2.7$ & $33.84 \pm 2.0$ & -2.809 & $0.005^{*}$ \\
\hline & Dig. Tot. & Media \pm D.T. & $13.68 \pm 3.4$ & $14.56 \pm 3.4$ & -2.586 & $0.008 \%$ \\
\hline & Dig. Dir. & Media \pm D.T. & $8.38 \pm 2.0$ & $8.62 \pm 2.0$ & -0.843 & 0.399 \\
\hline & Dig. In. & Media \pm D.T. & $5.47 \pm 2.3$ & $6.06 \pm 1.7$ & -2.641 & $0.008 *$ \\
\hline & Mem. Mot. & Media \pm D.T. & $6.78 \pm 2.4$ & $8.26 \pm 1.9$ & -3.306 & $0.001 \%$ \\
\hline & Orient. Tot. & Media \pm D.T. & $12.03 \pm 3.8$ & $13.93 \pm 2.8$ & -3.187 & $0.001 \%$ \\
\hline & Orient. 1 & Media \pm D.T. & $6.24 \pm 1.9$ & $7.07 \pm 1.4$ & -1.819 & 0.069 \\
\hline & Orient. 2 & Media \pm D.T. & $5.79 \pm 2.2$ & $6.82 \pm 1.7$ & -3.130 & $0.002 *$ \\
\hline \multirow{8}{*}{$\begin{array}{l}\text { Universitarios } \\
(\mathrm{n}=14)\end{array}$} & MEC & Media \pm D.T. & $32.93 \pm 1.8$ & $34.07 \pm 1.3$ & -1.864 & 0.065 \\
\hline & Dig. Tot. & Media \pm D.T. & $14.14 \pm 3.4$ & $15.57 \pm 3.2$ & -1.894 & 0.058 \\
\hline & Dig. Dir. & Media \pm D.T. & $8.36 \pm 1.6$ & $8.29 \pm 2.7$ & -0.540 & 0.589 \\
\hline & Dig. In. & Media \pm D.T. & $5.79 \pm 2.4$ & $6.57 \pm 1.7$ & -1.725 & 0.084 \\
\hline & Mem. Mot. & Media \pm D.T. & $6.77 \pm 2.8$ & $8.79 \pm 1.6$ & -2.831 & $0.005^{*}$ \\
\hline & Orient. Tot. & Media \pm D.T. & $12.21 \pm 2.8$ & $14.57 \pm 1.7$ & -2.523 & $0.012 \%$ \\
\hline & Orient. 1 & Media \pm D.T. & $6.43 \pm 1.6$ & $7.43 \pm 1.2$ & -1.647 & 0.100 \\
\hline & Orient. 2 & Media \pm D.T. & $5.86 \pm 1.9$ & $7.14 \pm 1.0$ & -2.435 & $0.015 \%$ \\
\hline
\end{tabular}


Tabla 9 - Análisis descriptivo e inferencia estadística de las puntuaciones obtenidas en pre-post test del grupo experimental según nivel de estudios. Pontevedra, España, 2010. (Continuação)

\begin{tabular}{|c|c|c|c|c|c|c|}
\hline \multicolumn{7}{|c|}{ Grupo experimental $(\mathrm{n}=185)$} \\
\hline & & & \multirow[b]{2}{*}{ Pretest } & \multirow[b]{2}{*}{ Postest } & \multicolumn{2}{|c|}{ P. Wilcoxon } \\
\hline & & & & & $\mathrm{Z}$ & $\begin{array}{c}\text { Valor de } \\
\text { Significación }\end{array}$ \\
\hline \multirow{8}{*}{$\begin{array}{l}\text { Analfabeto/a } \\
(\mathrm{n}=7)\end{array}$} & MEC & Media \pm D.T. & $25.21 \pm 4.0$ & $29.00 \pm 1.8$ & -2.023 & $0.043 *$ \\
\hline & Dig. Tot. & Media \pm D.T. & $8.43 \pm 1.6$ & $10.00 \pm 3.0$ & -1.214 & 0.225 \\
\hline & Dig. Dir. & Media \pm D.T. & $5.29 \pm 1.1$ & $6.00 \pm 1.4$ & -1.289 & 0.197 \\
\hline & Dig. In. & Media \pm D.T. & $3.14 \pm 1.0$ & $4.00 \pm 1.9$ & -0.649 & 0.516 \\
\hline & Mem. Mot. & Media \pm D.T. & $6.00 \pm 2.5$ & $4.43 \pm 3.6$ & -0.765 & 0.444 \\
\hline & Orient. Tot. & Media \pm D.T. & $5.67 \pm 3.0$ & $10.33 \pm 3.4$ & -2.023 & $0.043^{*}$ \\
\hline & Orient. 1 & Media \pm D.T. & $3.83 \pm 1.4$ & $5.83 \pm 1.9$ & -1.826 & 0.068 \\
\hline & Orient. 2 & Media \pm D.T. & $1.83 \pm 2.1$ & $4.50 \pm 1.6$ & -2.032 & $0.042 *$ \\
\hline
\end{tabular}

Dig Tot.: Dígitos Total; Dig. Dir: Dígitos Directo; Dig. In.: Dígitos Inverso; Mem. Mot.: Prueba Memoria Motriz; Orient. Tot.: Prueba Orientación Total; Orient. 1: Prueba Orientación 1; Orient. 2: Prueba Orientación 2 $\mathrm{p}<0.05 \%$ (La diferencia es significativa al nivel de 0.05 )

De la tabla 9 se desprenden los siguientes resultados del grupo experimental según el nivel de estudios: Mejoras significativas $(\leq 0.05)$ de las personas con estudios primarios en todos los valores excepto en Dígitos Directo e Inverso. Mejoras significativas $(\leq 0.05)$ de las personas con estudios secundarios en todos los valores excepto Dígitos Directo y Orientación Espacial 1. Mejoras significativas $(\leq 0.05)$ de las personas con estudios universitarios en Memoria Motriz, Orientación Espacial Total y Espacial 2. Mejoras significativas $(\leq 0.05)$ de las personas analfabetas en MEC, Orientación Total y Orientación 2. Por lo tanto, los sujetos del grupo experimental con estudios primarios y secundarios mejoran más significativamente que los sujetos con estudios universitarios y sin estudios.

\section{DISCUSIÓN}

Los resultados expuestos descubren mejoras cognitivas en el grupo experimental tras su participación en el programa "Memoria en Movimiento" y coinciden con otras investigaciones que señalan mejoras significativas en las mediciones de las funciones cognitivas tras la aplicación de programas de memoria. ${ }^{7,25,26}$

Tanto los trabajos anteriores como aquellos que incorporan programas mixtos - entrenamiento cognitivo y entrenamiento físico $-^{5,12,13}$ o programas integrados - entrenamiento cognitivomotriz $-{ }^{11}$ corroboran los resultados de la presente investigación y ratifican la importancia de programas específicos para la mejora de las funciones cognitivas.

Fabre et al. ${ }^{13}$ establecieron que la combinación de entrenamiento mental y aeróbico proporciona un efecto cognitivo mayor que cualquiera de los dos entrenamientos por separado. Aunque el presente estudio no puede ratificar tales conclusiones porque no incluyó en el diseño un grupo experimental sometido exclusivamente a entrenamiento físico o mental, los resultados de esta investigación coinciden con las mejoras cognitivas obtenidas por el grupo experimental 3 - entrenamiento aeróbico + mental - de Fabre et al. ${ }^{13}$

A pesar de esta correlación entre ambos estudios, existen grandes diferencias entre el entrenamiento aeróbico-mental desarrollado por Fabre et al. ${ }^{13}$ y el programa "Memoria en Movimiento". ${ }^{17} \mathrm{El}$ primero incluye módulos independientes de entrenamiento aeróbico y entrenamiento mental mientras que "Memoria en movimiento" integra en el mismo módulo y en todas las tareas el trabajo cognitivo con el motriz.

Precisamente, es esta línea de indagación la que avala el programa de intervención. La interacción 
del trabajo perceptivo-motriz con mejoras en la memoria es lo más novedoso y relevante de este trabajo.

En la misma línea se encuentra el trabajo realizado por Pont,$^{11}$ que confirma que para la mejora de la memoria motriz es preferible integrar el entrenamiento motriz con el cognitivo frente a la práctica exclusiva de ejercicio físico. Los resultados que obtiene esta autora en su investigación después de aplicar un entrenamiento integral cognitivo-motriz concuerdan con las mejoras obtenidas por el grupo experimental de "Memoria en Movimiento" en la Prueba de Memoria Motriz.

En cuanto a las diferencias significativas de las puntuaciones iniciales en la Prueba de Memoria Motriz de los dos grupos pueden estar causadas porque las personas del grupo control eran activas físicamente - participaban desde hacía cinco meses en actividades de gimnasia y/o tai chi -. Por su parte, los integrantes del grupo experimental eran inactivos físicamente al comienzo de las primeras mediciones. Dado que la práctica regular de actividad física preserva la propiocepción, ${ }^{4,14,27}$ puede haber influido en la evaluación al tratarse de una prueba de memoria a corto plazo que requiere de información espacial, en la que la superposición de informaciones - táctil y kinestésica - es particularmente importante. ${ }^{28}$ Con todo, las mejoras en el grupo experimental después de su participación en el programa en contraposición a los resultados del grupo control se han hecho patentes.

En cuanto a las pruebas de Orientación Espacial, las mejoras significativas apreciadas en el grupo experimental concuerdan con los resultados de Calero y García. $^{29}$ Los entrenamientos de aptitudes espaciales específicos que implican solución de problemas próximos a la vida real obtienen resultados positivos. Diferentes estudios sugieren efectos beneficiosos del entrenamiento sobre el deterioro de la memoria espacial asociado a la edad, ya que la plasticidad cerebral induciría cambios neurológicos. ${ }^{30}$
Cuando se lleva a cabo el análisis intragrupos del grupo experimental según intervalos de edad, se observa que en todos los valores iniciales los resultados son dispares, lo que no permite admitir una relación directa edad-nivel de función cognitiva. Diversos autores ${ }^{31,32}$ apuntan que el funcionamiento cognitivo no se correlaciona directamente con un único factor, sino que es sistémico y susceptible de la influencia combinada de varios factores: edad, nivel de estudios, género y estilos de vida. Esta confluencia idiosincrásica de cada individuo podría explicar los resultados dispares edad-rendimiento cognitivo.

El análisis intragrupos del factor nivel de estudios del grupo experimental permite contrastar los mejores rendimientos en los valores iniciales de los sujetos con mayor nivel de estudios. Estos resultados coinciden con Schönknecht et al..$^{33}$ en cuanto que los niveles educativos determinan el aumento de la capacidad de reserva cognitiva, demorando la aparición del declinar cognitivo. Gómez y Ostrosky ${ }^{34}$ confirman la hipótesis de la reserva cognitiva, pero se muestran prudentes ante la compleja relación entre la educación y la capacidad cognitiva asociada a la edad.

De forma global, los datos obtenidos sugieren que, cuando las personas mayores participan en un programa de estimulación cognitiva a través de tareas que integran el trabajo cognitivo y motriz se producen mejoras importantes en el funcionamiento cognitivo general; en la atención, la memoria de trabajo, la memoria motriz, la conciencia corporal y la orientación espacial.

Además, el análisis intragrupos apunta mayores progresos en las personas de edades comprendidas entre los 50 y los 79 años, con estudios primarios o secundarios. Se trata de un primer avance que requiere de verificación en posteriores investigaciones.

\section{CONCLUSIONES}

"Memoria en Movimiento" es un programa de estimulación cognitiva para personas mayores que integra el entrenamiento cognitivo y 
perceptivo-motriz mediante el desarrollo de tareas que implican simultáneamente la cognición y la motricidad. Las tareas son sencillas, significativas y evitan el factor velocidad, pero con una demanda elevada del control ejecutivo. Los destinatarios/ as que obtienen los mayores beneficios de la participación en el programa son personas mayores de 60 años, sin deterioro cognitivo, sin problemas de movilidad y sin institucionalizar. El programa "Memoria en Movimiento" consigue a través de 26 sesiones el desarrollo de la atención, la memoria y las funciones perceptivo-motrices

\section{REFERENCIAS}

1. Park DC, Polk TA, Mikels JA, et al. Cerebral aging: integration of brain and behavioral models of cognitive function. Dialogues in clinical neurosciencie 2001; 3 (3): 151-165.

2. Sorel $\mathrm{O}$, Pennequin V. Aging of the planning process: the role of executive functioning. Brain and Cognition 2008; 66: 196-204.

3. Duarte JA, Appell HJ. Skeletal muscle and aging. European review of aging and physical activity 2007; 4 (1): 51-52.

4. Ribeiro F, Oliveira J. Aging effects on joint propioception: the role of physical activity in propioception. European Review of aging and Phisical Activity 2007; 4 (2): 71-76.

5. Fernández-Ballesteros R, Caprara MG, Iñiguez J, García LF. Promoción del envejecimiento activo: efectos del programa "Vivir con vitalidad”. Arch Gerontol Geriatrics 2005; 20 : 135-148.

6. Richeson N, Boyne S, Brady EM. Education for older adults with early-stage dementia: health promotion for the mind, body and spirit. Educ Gerontol 2007; 33: 723-736.

7. Belleviye $S$, et al. Improvement of episodic memory in persons with mild cognitive Impairment and healthy older adults, evidence from a cognitive intervention program. Dementia and geriatrics disorders 2006; 22: 5-6.

8. Zamarrón MD, Tárraga L, FernándezBallesteros R. Plasticidad cognitiva en personas con la enfermedad de Alzheimer que reciben programas de estimulación cognitiva. Psicothema 2008; 20(3): 432-437.

9. Kramer A, Erickson K, Colcombe S. Exercise, cognition, and the aging brain. J of Applied Physiology 2006; 101: 1237-1242. de la conciencia corporal y de la estructuración espacial de las personas mayores a partir de tareas de carácter lúdico y social que requieren la implicación motriz de los participantes.

\section{AGRADECIMIENTOS}

A todos los alumnos/as de "Memoria en Movimiento" por su participación. A la Obra Social Caixa Galicia por su apoyo y financiación -C0-015-07-.

10. Fragoso F, et al. Efecto del entrenamiento de fuerza sobre la autonomía funcional en mujeres mayores sanas. Rev Española de Geriatría y Gerontología 2007; 42(6): 342-347.

11. Pont $P$. Efectes d'un programa d'activitat física sobre la mèmoria en la gent gran.

Barcelona.Tesis [ doctoral en salud]Universitat de Barcelona; 2005.

12. Rebok GW, Plude DJ. Relation of physical activity to memory functioning in older adults, the memory workout program. Educ Gerontol 2001; 27 (3): 241-259.

13. Fabre $\mathrm{C}$, et al. Improvement of cognitive function by mental and/or individualizad aerobic training in healthy elderly subjects. Int J Sports Med 2002; 23(6): 415-421.

14. Kimura N, et al. Memory and physical mobility in physically and cognitively-independent elderly people. Geriatric and Gerontologist International 2007; 7: 258-265.

15. Schäfer S, Huxhold $O$, Lindenberger U. Healthy mind in healthy body: a review of sensorimotorcognitive interdependencies in old age. European Review of Aging and Physical Activity 2006; 3 (2): 45-54.

16. Dishman R, et al. Neurobiology of exercise. Obesity 2006; 14 (3): 345-356.

17. Rey A, CanalesI .Memoria en Movimiento: programa de estimulación cognitiva a través de la motricidad para personas mayores. Santiago de Compostela: 1 Caixa Galicia; 2008. [ Acceso en sep 2009]. Disponible en: http:// www.obrasocialcaixagalicia.org.

18. Anguera MT, et al. Métodos de investigación en psicología. Madrid: síntesis; 1995. 
19. Salthouse T, et al. Sources of individual differences in spatial visualization ability.Intelligence 1990; 14: 187-230.

20. Sclan SG, Reisberg MD. Functional assessment staging (FAST) in Alzheimer's disease: realiability, validity, and ordinality. International psychogeriatric 1992; 4 (1): 55-69.

21. Organización mundial de la salud. Clasificación Internacional del Funcionamiento de la Discapacidad y de la Salud: CIF. Madrid: Ministerio de Trabajo y Asuntos Sociales. Secretaría General de Asuntos Sociales; 2001

22. Lobo A, et al. Revalidación y normalización del Mini-Examen cognoscitivo (primera versión en castellano del Mini-Mental Status Examination) en la población general geriátrica. Interpsiquis 2001 feb. [ Acceso 3 dic 2007]; URL disponible en: http//:www.psiquiatria.com/psicología.

23. Seisdedos N. WAIS-III: manual técnico. Madrid: TEA; 1999.

24. Feuerstein R. Programa de enriquecimiento instrumental. Un momento déjame pensar. Madrid: Bruño Instituto S. Pío X Agregado; 2000.

25. Calero MD, Navarro E. Eficacia de un programa de entrenamiento en memoria en el mantenimiento de ancianos con y sin deterioro cognitivo. Clínica y Salud 2005; 17(2): 187-202.

26. Recio P, Rodríguez R, García MV. Mejora de la memoria en un caso mediante aprendizaje de estrategias nemotécnicas. Psicothema 2000; 12(2): 496-499.

27. Mulder TH, Hochstenbach JBH, van Heuvelen MJG, den Otter AR. Motor imagery, the relation between age and imagery capacity. Human movement Science 2007; 26: 203-211.

28. Millar S. Memory in touch. Psicothema 1999; 11 (4): 747-767.

29. Calero MD, García T. Entrenamiento de la competencia espacial en ancianos. Anuario de psicología 1995; 64: 67-81.

30. Vicens P, Redolat R, Carrasco MC. Aprendizaje espacial y laberinto de agua: metodología y aplicaciones. Psicothema 2003; 15 (4): 539-544.

31. Laursen $P$. The impact of aging on cognitive functions. An 11 year follow-up study of four aged cohorts. Acta Neurologica Scandinavica 1997; 96: 1-86.

32. Martín M, Moreno P, Blas J, Buelbena A. Deterioro cognitivo ligero. Geriátrika 2004; 20 (2): 58-71.

33. Schönknecht P, Pantel J, Kruse A, Schröder J. Prevalence and natural course of agingassociated cogntive decline in a populationbased sample o young old subjects. Am J Psychiatry 2005; 162: 2071-2077.

34. Gomez E, Ostrosky F. Attention and memory evaluation across the Life Span, Heterogeneous effects of age and education. J of Clinical and Exp Neuropsychol 2006; 28: 477-494. 
